\title{
Decentralized Decision-Making Over Multi-Task Networks
}

\author{
Sahar Khawatmi, Abdelhak M. Zoubir ${ }^{1}$ \\ Technische Universität Darmstadt, Signal Processing Group \\ 64283 Darmstadt, Germany \\ Email: \{khawatmi, zoubir\}@spg.tu-darmstadt.de \\ Ali H. Sayed ${ }^{2}$ \\ Ecole polytechnique fédérale de Lausanne EPFL, Adaptive Systems Laboratory \\ CH-1015 Lausanne, Switzerland \\ Email: ali.sayed@epfl.ch
}

\begin{abstract}
In important applications involving multi-task networks with multiple objectives, agents in the network need to decide between these multiple objectives and reach an agreement about which single objective to follow for the network. In this work we propose a distributed decision-making algorithm. The agents are assumed to observe data that may be generated by different models. Through localized interactions, the agents reach agreement about which model to track and interact with each other in order to enhance the network performance. We investigate the approach for both static and mobile networks. The simulations illustrate the performance of the proposed strategies.

Keywords: Decentralized processing, decision-making, multi-task networks, adaptive learning.
\end{abstract}

\section{Introduction and Related Work}

Bio-inspired systems are designed to mimic the behavior of some animal groups such as bee swarms, birds flying in formation, and schools of fish $[1,2,2,3,4,4$,

\footnotetext{
${ }^{1}$ Member of the European Association for Signal Processing (EURASIP).

${ }^{2}$ Member of the European Association for Signal Processing (EURASIP).
}

Preprint submitted to EURASIP signal processing journal 
6]. Diffusion strategies can be used to model some of these coordinated types of behavior, as well as solve inference and estimation tasks in a distributed manner over networks [7, 8]. We may distinguish between two types of networks: single-task and multi-task networks. In single-task implementations [7, 8], the networks consist of agents that are interested in the same objective and sense data that are generated by the same model. An analogy would be a school of fish tracking a food source: all elements in the fish school sense distance and direction to the same food source and are interested in approaching it. On the other hand, multi-task networks [9, 10, 11, 12, 13, 14, 15, 16, 17] involve agents sensing data arising from different models and different clusters of agents may be interested in identifying separate models. A second analogy is a school of fish sensing information about multiple food sources.

In the latter case, agents need to decide between the multiple objectives and reach agreement on following a single objective for the entire network. In the earlier works [18, 19], a scenario was considered where agents were assumed to sense data arising from two models, and a diffusion strategy was developed to enable all agents to agree on estimating a single model. The algorithm developed in 18] relied on binary labeling and is applicable only to situations involving two models. In this work, we propose an approach for more than two models.

We consider a distributed mean-square-error estimation problem over an $N$ agent network. The connectivity of the agents is described by a graph (see Fig. 11). Data sensed by any particular agent can arise from one of different models. The objective is to reach an agreement among all agents in the network on one common model to estimate. Two definitions are introduced: the observed model, which refers to the model from which an agent collects data, and the desired model, which refers to the model the agent decides to estimate. The agents do not know which model generated the data they collect; they also do not know which other agents in their neighborhood sense data arising from the same model. Therefore, each agent needs to determine the subset of its neighbors that observes the same model. This initial step is referred to as clustering. Since the decision-making objective depends on the clustering output, errors made during 
the clustering process have an impact on the global decision. In this work, we rely on the clustering technique proposed in [20] to reduce this effect.

The paper is organized as follows. The network and data model are described in Section II. We illustrate the local labeling system and the decision-making algorithm in Sections III and IV, respectively. A special case when the entire network follows the model of a specific agent is studied in Section V. Simulation results and discussion are presented in Sections VI and VII, respectively.

Notation. We use lowercase letters to denote vectors, uppercase letters for matrices, plain letters for deterministic variables, and boldface letters for random variables. $\mathbb{E}$ denotes the expectation operator and $\|\cdot\|$ the Euclidean norm. The symbols $\mathbb{1}$ and $I$ denote the all-one vector and identity matrix of appropriate sizes, respectively. The $k$-th row (column) of matrix $X$ is denoted by $[X]_{k, \text { : }}$ $\left([X]_{:, k}\right)$.

\section{Network and Data Model}

Consider a collection of $N$ agents distributed in space, as illustrated in Fig. 1 We represent the network topology by means of an $N \times N$ adjacency matrix $E$ whose entries $e_{\ell k}$ are defined as follows:

$$
e_{\ell k}= \begin{cases}1, & \ell \in \mathcal{N}_{k}, \\ 0, & \text { otherwise }\end{cases}
$$

where $\mathcal{N}_{k}$ is the set of neighbors of agent $k$ (we denote its size by $n_{k}$ ). We also write $\mathcal{N}_{k}^{-}$to denote the same neighborhood excluding agent $k$.

Figure 1 shows the network structure where agents with the same color observe the same model. We denote the unknown models by $\left\{z_{1}^{\circ}, \ldots, z_{C}^{\circ}\right\}$, each of size $M \times 1$ where $C \leq N$. Each agent $k$ observes data generated by one of these $C$ unknown models. We denote the model observed by agent $k$ by $w_{k}^{\circ}$. Figure1 shows that agent $k$ collects data from model $z_{1}^{\circ}$, in which case $w_{k}^{\circ}=z_{1}^{\circ}$. For any other agent $\ell$ observing the same model $z_{1}^{\circ}$, it will hold that $w_{\ell}^{\circ}=z_{1}^{\circ}$. 
We stack the $\left\{w_{k}^{\circ}\right\}$ into a column vector:

$$
w^{\circ} \triangleq \operatorname{col}\left\{w_{1}^{\circ}, w_{2}^{\circ}, \cdots, w_{N}^{\circ}\right\}, \quad w^{\circ} \in \mathbb{R}^{M N \times 1} .
$$

At every time instant $i$, every agent $k$ has access to a scalar measurement $\boldsymbol{d}_{k}(i)$ and a $1 \times M$ regression vector $\boldsymbol{u}_{k, i}$. The measurements across all agents are assumed to be generated via linear regression models of the form:

$$
\boldsymbol{d}_{k}(i)=\boldsymbol{u}_{k, i} w_{k}^{\circ}+\boldsymbol{v}_{k}(i)
$$

All random processes are assumed to be stationary. Moreover, $\boldsymbol{v}_{k}(i)$ is a zeromean white measurement noise that is independent over space and has variance $\sigma_{v, k}^{2}$. The regression data $\boldsymbol{u}_{k, i}$ is assumed to be a zero-mean random process, independent over time and space, and independent of $\boldsymbol{v}_{\ell}(j)$ for all $k, \ell, i, j$. We denote the covariance matrix of $\boldsymbol{u}_{k, i}$ by $R_{u, k}=\mathbb{E} \boldsymbol{u}_{k, i}^{\top} \boldsymbol{u}_{k, i}$.

Agents do not know which model is generating their data. They also do not know which models are generating the data of their neighbors. Still, we would like to perform a learning strategy that allows agents to converge towards one of the models, while also learning which of their neighbors share the same model. Using the algorithm proposed in 20], each agent $k$ repeats the following steps involving an adaptation step followed by an aggregation step:

$$
\begin{aligned}
\boldsymbol{\psi}_{k, i} & =\boldsymbol{\psi}_{k, i-1}+\mu_{k} \boldsymbol{u}_{k, i}^{\top}\left(\boldsymbol{d}_{k}(i)-\boldsymbol{u}_{k, i} \boldsymbol{\psi}_{k, i-1}\right) \\
\boldsymbol{\phi}_{k, i} & =\sum_{\ell=1}^{N} \boldsymbol{a}_{\ell k}(i) \boldsymbol{\psi}_{\ell, i}
\end{aligned}
$$

where $\mu_{k}$ is the step-size used by agent $k$. These updates generate two iterates by agent $k$ at time $i$, and which are denoted by $\boldsymbol{\psi}_{k, i}$ and $\phi_{k, i}$, respectively. We collect the estimated vectors across all agents into the aggregate vector:

$$
\phi_{i} \triangleq \operatorname{col}\left\{\phi_{1, i}, \phi_{2, i}, \cdots, \phi_{N, i}\right\}
$$

In a manner similar to [20], we introduce a clustering matrix $\boldsymbol{E}_{i}$. Its structure is similar to the adjacency matrix $E$, with ones and zeros, except that the value at location $(\ell, k)$ will be set to one if agent $k$ believes at time instant $i$ that its 


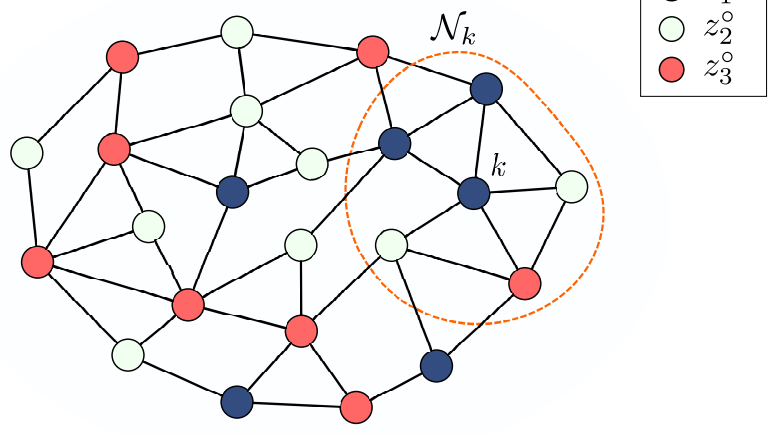

Figure 1: Example of a network topology, agents with the same color observe the same model.

neighbor $\ell$ belongs to the same cluster, i.e., observes the same model:

$$
\boldsymbol{e}_{\ell k}(i)= \begin{cases}1, & \text { if } \ell \in \mathcal{N}_{k} \text { and } k \text { believes that } w_{k}^{\circ}=w_{\ell}^{\circ} \\ 0, & \text { otherwise }\end{cases}
$$

These entries help define the neighborhood set $\mathcal{N}_{k, i}$, which consists of all neighbors at time instant $i$ that agent $k$ believes share the same model. To learn the matrix $\boldsymbol{E}_{i}$ over time, we apply the clustering technique proposed in [20] to create the estimated clustering matrix $\boldsymbol{F}_{i}$ of size $N \times N$ as follows: we initialize $\boldsymbol{\psi}_{k,-1}=0$ and $\boldsymbol{B}_{-1}=\boldsymbol{F}_{-1}=\boldsymbol{E}_{-1}=I_{N}$. Where the matrix $\boldsymbol{B}_{i}$ is of size $N \times N$. Each entry $\boldsymbol{e}_{\ell k}(i)$ is designed using the following steps from [20], where $\ell \in \mathcal{N}_{k}:$

$$
\begin{gathered}
\boldsymbol{b}_{\ell k}(i)=\left\{\begin{array}{l}
1, \text { if }\left\|\boldsymbol{\psi}_{\ell, i}-\boldsymbol{\phi}_{k, i-1}\right\|^{2} \leq \alpha \\
0, \text { otherwise }
\end{array}\right. \\
\boldsymbol{f}_{\ell k}(i)=\nu \times \boldsymbol{f}_{\ell k}(i-1)+(1-\nu) \times \boldsymbol{b}_{\ell k}(i)
\end{gathered}
$$

where $\alpha>0,0 \leq \nu \leq 1$, and the notation $\lfloor\cdot\urcorner$ denotes rounding to the nearest integer. Using the evolving neighborhoods $\mathcal{N}_{k, i}$, the entries $\left\{\boldsymbol{a}_{\ell k}(i)\right\}$ in (5) are 
non-negative scalars that satisfy

$$
\boldsymbol{a}_{\ell k}(i)=0 \text { for } \ell \notin \mathcal{N}_{k, i}, \quad \sum_{\ell=1}^{N} \boldsymbol{a}_{\ell k}(i)=1 .
$$

Although there is a multitude of models generating the data that is feeding into the agents, namely, $\left\{z_{1}^{\circ}, z_{2}^{\circ}, \ldots, z_{C}^{\circ}\right\}$, the objective is to develop a strategy that will allow all agents to converge towards one of these models. We refer to this particular choice as the desired model and denote it by $z_{d}^{\circ}$.

In this way, an agent whose source (observed) model agrees with the desired model, i.e., $w_{k}^{\circ}=z_{d}^{\circ}$, will end up tracking its own source. On the other hand, an agent whose source model is not the desired model, i.e., $w_{k}^{\circ} \neq z_{d}^{\circ}$, will track $z_{d}^{\circ}$ instead although it is sensing data generated by a different model.

We define the estimate vector of agent's $k$ desired model by $\boldsymbol{w}_{k, i}$. The reason behind indicating $\boldsymbol{w}_{k, i}$ as the estimate vector of agent's $k$ desired model instead of the network's desired model is that the agents may have different desired models before convergence (steady-state). Once the agents reach agreement among themselves on a single model, we can then refer to $\boldsymbol{w}_{k, i}$ as the estimate vector by agent $k$ of the network's desired model. For the initialization at time instant $i=1$, each agent assigns $\boldsymbol{w}_{k, 0}=\boldsymbol{\psi}_{k, 1}$ (i.e., at time instant $i=1$, the desired model of each agent is a rough estimate of its own source model). The decision-making process drives the desired models of all agents to converge. For example, if the agents observe $C=5$ different models, the number of the desired models in the network will decrease with iterations gradually form 5 models down to one model. This is achieved by switching the estimate $\boldsymbol{w}_{k, i}$ of some agents during the decision-making process according to some conditions that are explained later. However, agents do not know which models are desired by their neighbors at each time instant $i$. Thus, we need to develop a learning strategy that allows the agents to distinguish the individual desired models of their neighbors.

It turns out that in order for the objective of the network to be met, it is important for agents to combine the estimates of their neighbors in a judicious manner because, unbeknown to the agents, some of their neighbors may be 
wishing to estimate different models. If cooperation is performed blindly with all neighbors, then performance can deteriorate with agents converging to nonexisting locations. For this reason, and motivated by the discussion from [18], we add the step (12) below after (4) and (5), which involves two sets of combination coefficients from two matrices $\dot{\boldsymbol{A}}_{i}$ and $\ddot{\boldsymbol{A}}_{i}$. There are two main ideas behind the construction (12). First, it is meant to let each agent $k$ cooperate only with the subset of neighbors that share the same desired model as it does. Second, it also lets each agent $k$ combine $\phi_{\ell, i}$ if the desired model of agent $k$ at time instant $i$ is the same as $\ell$ 's observed model:

$$
\boldsymbol{w}_{k, i}=\sum_{\ell=1}^{N} \dot{\boldsymbol{a}}_{\ell k}(i) \phi_{\ell, i}+\sum_{\ell=1}^{N} \ddot{\boldsymbol{a}}_{\ell k}(i) \boldsymbol{w}_{\ell, i-1} .
$$

Note that the matrices $\dot{\boldsymbol{A}}_{i}$ and $\ddot{\boldsymbol{A}}_{i}$ are not constructed from matrix $\boldsymbol{A}_{i}$. The selection of the non-negative coefficients $\left\{\dot{\boldsymbol{a}}_{\ell k}(i)\right\}$ and $\left\{\ddot{\boldsymbol{a}}_{\ell k}(i)\right\}$ is explained in Section IV.

We summarize the main five steps of the approach:

1. Learning the observed models of the neighbors. This step is performed by building the matrix $\boldsymbol{E}_{i}$ in step (7). The information provided by each entry $\boldsymbol{e}_{\ell k}(i)$ is whether the corresponding agents $\ell$ and $k$ have the same observed model or not.

2. Learning and labeling the desired model of the neighbors at each time instant $i$. This step allows the agents to distinguish the individual desired models of their neighbors at time instant $i$. The information provided by this step is the number of different models that are desired by neighbors and how many times each model is repeated at time $i$ among neighbors.

3. Decision-making step by switching the desired model of some agents to let the network converge to only one model.

4. Learning the desired models of the neighbors after the switching step. This step is performed by building the matrix $\boldsymbol{H}_{i}$ in step (19) in Section IV. The information provided by each entry $\boldsymbol{h}_{\ell k}(i)$ is whether the corresponding agents $\ell$ and $k$ have the same desired model or not after the switching step. 


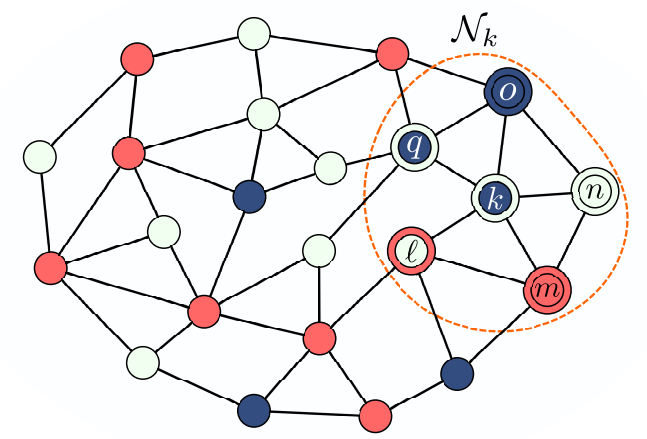

Figure 2: Example of an agent $k$ and its neighborhood $\mathcal{N}_{k}$. The inner color indicates the observing model while the outer one indicates the current desired model.

5. Updating the estimate vectors $\left\{\boldsymbol{w}_{k, i}\right\}$ by sharing data thoughtfully with the subset of the neighbors that share the same desired model.

\section{Local Labeling}

Each agent needs to learn the desired models of its neighbors to proceed with the decision-making process and let the network converge to only one model. In this step, instead of only estimating whether two agents have the same desired model or not, the construction involves a local labeling procedure that enables every agent to estimate in real-time how many different models are desired by its neighborhood.

For this purpose, we associate with each agent $k$ an $n_{k} \times n_{k}$ matrix $\boldsymbol{Y}_{i}^{k}$ with entries $\left\{\boldsymbol{y}_{\ell m}^{k}(i)\right\}$ given by:

$$
\boldsymbol{y}_{\ell m}^{k}(i)= \begin{cases}1, & \text { if }\left\|\boldsymbol{w}_{m, i-1}-\boldsymbol{w}_{\ell, i-1}\right\|^{2} \leq \beta \\ 0, & \text { otherwise }\end{cases}
$$

for some small threshold $\beta>0$. Whenever $\boldsymbol{y}_{\ell m}^{k}(i)=1$, agent $k$ believes at time instant $i$ that its neighbors $\ell$ and $m$ wish to estimate the same desired model. On account of that, the variables $\boldsymbol{w}_{m, i-1}$ and $\boldsymbol{w}_{\ell, i-1}$ which are used in the test (13) are presenting the current desired model of agents $m$ and $\ell$, 
respectively. It is clear from (13) that the matrix $\boldsymbol{Y}_{i}^{k}$ is symmetric and has ones on the diagonal. An example is depicted in Fig. 2 where agents having the same inner color observe the same model, while the outer color indicates the model in which the agent is interested (or towards which the agent is moving in mobile networks). The corresponding matrix $\boldsymbol{Y}_{i}^{k}$ has the following entries:

$$
\boldsymbol{Y}_{i}^{k}=\begin{gathered}
k \\
\ell \\
n \\
o \\
q \\
0 \\
0
\end{gathered}\left[\begin{array}{llllll}
k & \ell & m & n & o & q \\
1 & 0 & 0 & 1 & 0 & 1 \\
0 & 1 & 1 & 0 & 0 & 0 \\
0 & 1 & 1 & 0 & 0 & 0 \\
1 & 0 & 0 & 1 & 0 & 1 \\
0 & 0 & 0 & 0 & 1 & 0 \\
1 & 0 & 0 & 1 & 0 & 1
\end{array}\right] .
$$

From (14) agents that share the same desired model will have identical columns in matrix $\boldsymbol{Y}_{i}^{k}$, namely, if agents $m$ and $\ell$ have the same desired model at time instant $i$, this implies that: $\left[\boldsymbol{Y}_{i}^{k}\right]_{:, m}=\left[\boldsymbol{Y}_{i}^{k}\right]_{:, \ell}$. We denote the local label of each agent $\ell \in \mathcal{N}_{k}$ by agent $k$ as $\boldsymbol{l}_{\ell}^{k}(i)$. The local label $\boldsymbol{l}_{\ell}^{k}(i)$ is updated at each time instant $i$ using the following relation:

$$
\boldsymbol{l}_{\ell}^{k}(i)=\mathcal{B}\left(\left[\boldsymbol{Y}_{i}^{k}\right]_{:, \ell}\right)
$$

where $\mathcal{B}(\cdot)$ is a function that converts the input sequence from binary to decimal. For the example in (14), we have

$$
\begin{aligned}
& \boldsymbol{l}_{k}^{k}(i)=\mathcal{B}(100101)=37, \\
& \boldsymbol{l}_{\ell}^{k}(i)=\mathcal{B}(011000)=24, \\
& \boldsymbol{l}_{m}^{k}(i)=\mathcal{B}(011000)=24, \\
& \boldsymbol{l}_{n}^{k}(i)=\mathcal{B}(100101)=37, \\
& \boldsymbol{l}_{o}^{k}(i)=\mathcal{B}(000010)=2, \\
& \boldsymbol{l}_{q}^{k}(i)=\mathcal{B}(100101)=37 .
\end{aligned}
$$

We define the number of desired models within $\mathcal{N}_{k}$ at time instant $i$ by $\boldsymbol{C}_{k}(i)$. After updating matrix $\boldsymbol{Y}_{i}^{k}$ and generating the local labels $\left\{\boldsymbol{l}_{\ell}^{k}(i)\right\}$, agent $k$ counts 
how many models are desired by its neighborhood to update $\boldsymbol{C}_{k}(i)$. In the example (14), agent $k$ distinguishes at time instant $i$ three desired models $\{2,24,37\}$, i.e., $\boldsymbol{C}_{k}(i)=3$. Agent $k$ labels these three different models locally as: $\{2,24,37\}$.

In addition, agent $k$ determines which model among these $\boldsymbol{C}_{k}(i)$ models has the maximum number of followers. A follower of a model is an agent that wishes to estimate and track this model. We define the largest set of agents belonging to $\mathcal{N}_{k}$ and following the same desired model at time instant $i$ by $\mathcal{Q}_{k, i}$. In the example, agent $k$ assigns the majority set at time instant $i$ as follows: $\mathcal{Q}_{k, i}=\{k, n, q\}$ which has the label 37 and is repeated three times among other labels.

\section{Decision-Making Over Multi-Task Networks}

Using the information provided by matrix $\boldsymbol{Y}_{i}^{k}$, agent $k$ can capture how many agents within its neighbors follow the same desired model at time instant $i$. Once agent $k$ and all its neighbors agree on a single desired model, the matrix $\boldsymbol{Y}_{i}^{k}$ will end up being of the following form with unit entries everywhere:

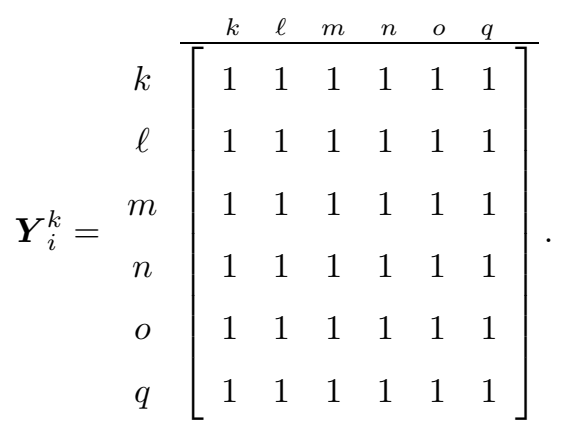

We define the degree of agreement by each agent $k$ among its neighbors $\mathcal{N}_{k}$ as

$$
\boldsymbol{p}_{k}(i)=\frac{\left[\boldsymbol{Y}_{i}^{k}\right]_{k,:} \mathbb{1}}{n_{k}} .
$$

Equally, having $\boldsymbol{p}_{k}(i)=1$ means that agent $k$ and all of its neighbors have agreed on a common desired model. On the other hand, if $\boldsymbol{p}_{k}(i) \neq 1$, then the 
following switching step is applied:

$$
\boldsymbol{w}_{k, i-1} \leftarrow \begin{cases}\boldsymbol{w}_{\ell, i-1}, & \text { if } k \notin \mathcal{Q}_{k, i} \text { for any } \ell \in \mathcal{Q}_{k, i}, \\ \boldsymbol{w}_{n, i-1}, & \text { if } k \in \mathcal{Q}_{k, i} \text { and } \boldsymbol{C}_{k}(i)=2, \forall n \in \mathcal{N}_{k}, \\ \boldsymbol{w}_{k, i-1}, & \text { otherwise. }\end{cases}
$$

The main idea of the switching step is for each agent $k$ to make a new decision or to keep the previous one. The first case of (18) implies that agent $k$ does not belong to the majority desired model set $\mathcal{Q}_{k, i}$ at time instant $i$. Therefore, agent $k$ changes its decision and switches into the desired model of the majority set $\mathcal{Q}_{k, i}$. The second case in (18) is applied to prevent an unwanted equilibrium situation. This problem may arise when only two desired models remain in $\mathcal{N}_{k}$. In this case, if all agents in $\mathcal{N}_{k}$ belong to the majority set, this leads to a situation in which no agent in $\mathcal{N}_{k}$ will change its decision anymore. An example is shown in Fig. 3 where the outer color of the agents indicate the desired model. We indicate only the desired model of agent's $k$ neighbors and their neighbors. Figure 3 shows that all agents within $\mathcal{N}_{k}$ belong to a majority set and no agent in $\mathcal{N}_{k}$ will change its decision anymore, e.g. agents $q$ and $\ell$ belong to the majority set among their neighbors, as well as agents $k, m, n$, and $o$. Namely,

$$
\begin{aligned}
& k \in \mathcal{Q}_{k, i}, m \in \mathcal{Q}_{m, i}, n \in \mathcal{Q}_{n, i} \text {, and } o \in \mathcal{Q}_{o, i} \quad\left(\text { with } z_{1}^{\circ}\right), \\
& \left.\ell \in \mathcal{Q}_{\ell, i} \text { and } q \in \mathcal{Q}_{q, i} \text { (with } z_{2}^{\circ}\right) \text {. }
\end{aligned}
$$

To break the equilibrium, an agent that recognizes these two models picks randomly one of the two desired models.

From (18), we can conjecture that the network will probably converge to the most observable model, since the initial desired model by each agent is its own observed model. This fact remains true even with the random switching in the second case of (18), because in that case the more repeated desired model within $\mathcal{N}_{k}$ has the highest probability to be picked.

To proceed with the cooperation and sharing information among the agents within the subset that has the same desired model at time instant $i$, we define an $N \times N$ matrix $\boldsymbol{H}_{i}$. The coefficients $\left\{\boldsymbol{h}_{\ell k}(i)\right\}$ are updated after the switching 


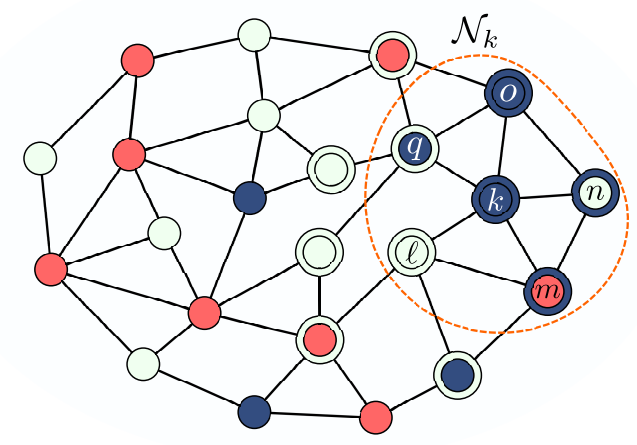

Figure 3: Example of the equilibrium case. All agents within $\mathcal{N}_{k}$ belong to the majority sets among their neighbors.

step (18) using a test that is quite similar to (13) and is applied between each agent $k$ and its neighbors as follows:

$$
\boldsymbol{h}_{\ell k}(i)= \begin{cases}1, & \text { if }\left\|\boldsymbol{w}_{k, i-1}-\boldsymbol{w}_{\ell, i-1}\right\|^{2} \leq \beta \\ 0, & \text { otherwise }\end{cases}
$$

According to matrix $\boldsymbol{H}_{i}$, each agent knows which subset of its neighbors has the same desired model as it does after the switching step at time instant $i$. Having $\boldsymbol{h}_{\ell k}(i)=1$ means that $\ell$ and $k$ have the same desired model at time instant $i$. We define an $N \times N$ combination matrix $\boldsymbol{G}_{i}$ as follows:

$$
\boldsymbol{G}_{i}=\mathcal{F}\left(\boldsymbol{H}_{i}\right)
$$

where $\mathcal{F}(\cdot)$ is some function which satisfies

$$
\boldsymbol{g}_{\ell k}(i)=0 \quad \text { if } \quad \boldsymbol{h}_{\ell k}(i)=0, \quad \sum_{\ell=1}^{N} \boldsymbol{g}_{\ell k}(i)=1
$$

An example of $\mathcal{F}(\cdot)$ is the uniform function which generates a left-stochastic matrix $\boldsymbol{G}_{i}$ where each entry $\boldsymbol{g}_{\ell k}(i)$ is given by

$$
\boldsymbol{g}_{\ell k}(i)= \begin{cases}\frac{1}{\sum_{n=1}^{N} \boldsymbol{h}_{n k}(i)}, & \text { if } \boldsymbol{h}_{\ell k}(i)=0, \\ 0, & \text { otherwise. }\end{cases}
$$


Matrix $\boldsymbol{G}_{i}$ by itself does not have enough information for proceeding and updating the estimate $\boldsymbol{w}_{k, i}$. The agents still need knowledge about which data to be combined from each neighbor. Therefore, matrix $\boldsymbol{G}_{i}$ is split into two matrices $\dot{\boldsymbol{A}}_{i}$ and $\ddot{\boldsymbol{A}}_{i}$. The weight of the entry $\boldsymbol{g}_{\ell k}(i)$ goes to $\dot{\boldsymbol{a}}_{\ell k}(i)$ if the desired model of agent $k$ at time instant $i$ is the same as $\ell$ 's observed model. Otherwise, $\ddot{\boldsymbol{a}}_{\ell k}(i)$ obtains the weight $\boldsymbol{g}_{\ell k}(i)$. The coefficients $\left\{\dot{\boldsymbol{a}}_{\ell k}(i)\right\}$ and $\left\{\ddot{\boldsymbol{a}}_{\ell k}(i)\right\}$ for $\ell \in \mathcal{N}_{k}$ are updated using the following steps:

$$
\begin{aligned}
& \dot{\boldsymbol{a}}_{\ell k}(i)= \begin{cases}\boldsymbol{g}_{\ell k}(i), & \text { if }\left\|\boldsymbol{w}_{k, i-1}-\boldsymbol{\psi}_{\ell, i}\right\|^{2} \leq \beta, \\
0, & \text { otherwise. }\end{cases} \\
& \ddot{\boldsymbol{a}}_{\ell k}(i)= \begin{cases}\boldsymbol{g}_{\ell k}(i), & \text { if } \dot{\boldsymbol{a}}_{\ell k}(i)=0, \\
0, & \text { otherwise. }\end{cases}
\end{aligned}
$$

In (23), the case that $\boldsymbol{\psi}_{\ell, i}$ is close to $\boldsymbol{w}_{\ell, i-1}$ implies that the observed model of agent $\ell$ is the same as the desired model of agent $k$ at time instant $i$. The estimate $\boldsymbol{w}_{k, i}$ is updated using (12). Algorithm 1 summarizes the decisionmaking scheme.

\section{Following the Observed Model of a Specific Agent}

In this section the goal is to let the whole network follow the observed model of some specific agent $m$, as shown in Fig. 4 where agent $m$ observes model $z_{3}^{\circ}$ (red), therefore, the network converges in a distributed manner to estimate the model $z_{3}^{\circ}$. The first step is to spread the $\boldsymbol{\psi}_{m, i}$ among agents and keep updating it over time. This step aims at having a copy (reference) of $\boldsymbol{\psi}_{m, i}$ by all agents in the network. Agents keep updating the copy of $\boldsymbol{\psi}_{m, i}$ for two reasons. First, to have a more accurate version of the vector $\boldsymbol{\psi}_{m, i}$, which indicates the desired model of the network. Second, to endow the algorithm to work in non-stationary situations, if drift is happening in agent m's model.

We denote the copy vector of $\boldsymbol{\psi}_{m, i}$ by agent $k$ by $\breve{\boldsymbol{\psi}}_{k, i}$ and refer to it as the anchor vector. Agents are informed beforehand about the index $m$ of the specific agent that they should follow. If $m \in \mathcal{N}_{k}$, this implies that agent $k$ 


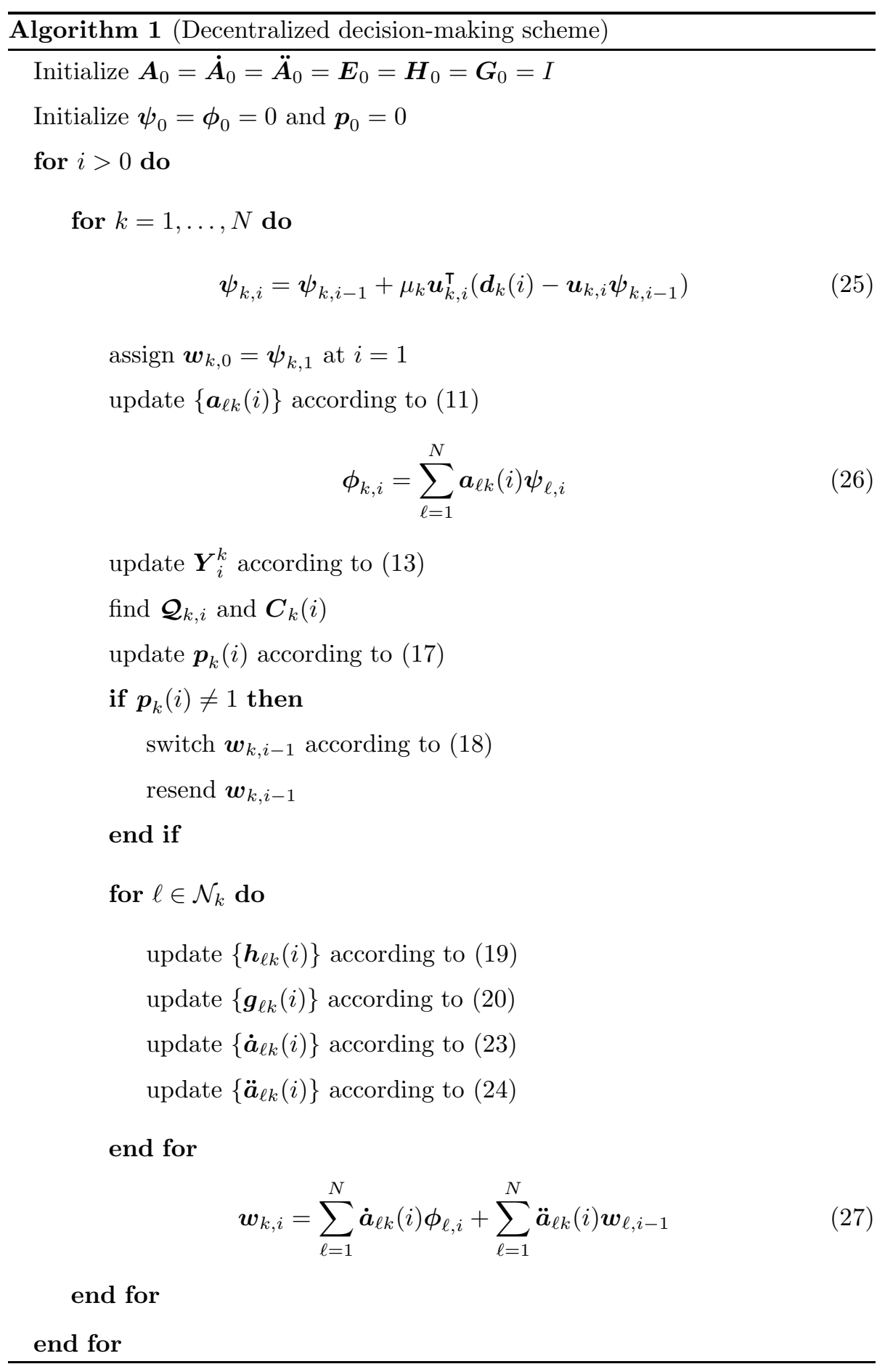




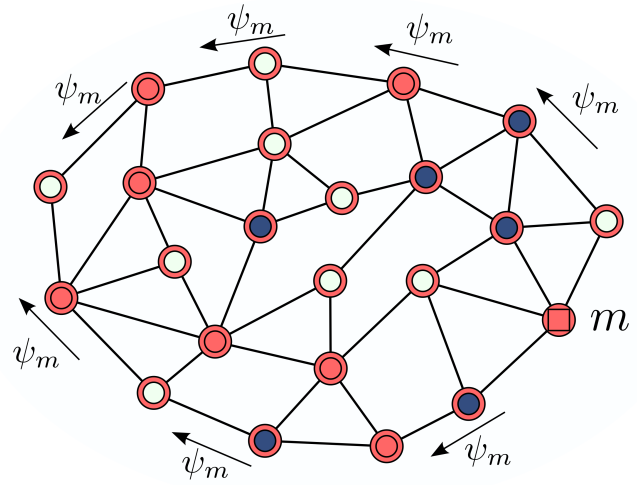

Figure 4: Final decision of a network after following the model of the specific agent $m$. The inner color indicates the observing model while the outer one indicates the desired model.

The arrows represent the spreading process of $\boldsymbol{\psi}_{m, i}$ through the network.

(a)

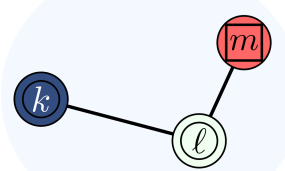

(b)

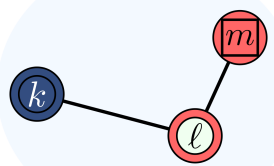

(c)

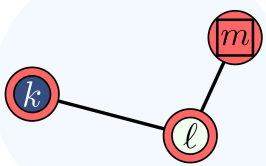

Figure 5: Example of the spreading process of $\boldsymbol{\psi}_{m, i}$ from agent $m$ to agent $k$ over time. The inner color indicates the observing model while the outer one indicates the desired model. 
receives the anchor vector directly from agent $m$. If not, i.e., $m \notin \mathcal{N}_{k}$, then agent $k$ depends on another agent $\ell \in \mathcal{N}_{k}$ that has already a copy of $\boldsymbol{\psi}_{m, i}$. Agent $k$ stores the index of this source agent. The index of the source agent of agent $k$ is denoted by $\boldsymbol{s}_{k}(i)$. Note that the anchor vector $\breve{\boldsymbol{\psi}}_{k, i}$ is not the final estimate of the desired model.

The circulation process of $\boldsymbol{\psi}_{m, i}$ in a distributed manner needs cooperation among agents. In case that agent $k$ has no direct link to receive data from agent $m$, i.e., $m \notin \mathcal{N}_{k}$, agent $k$ gets one of the $\breve{\boldsymbol{\psi}}_{\ell, i-1}$ provided that $\boldsymbol{s}_{\ell}(i) \neq 0$. If $\boldsymbol{s}_{\ell}(i) \neq 0$ this implies that agent $\ell$ has already a source to update its $\breve{\boldsymbol{\psi}}_{\ell, i}$, regardless whether $m \in \mathcal{N}_{\ell}$ or not. In other words, $s_{\ell}(i) \neq 0$ means that agent $\ell$ finds a direct or indirect link to agent $m$. Therefore, it is important for each agent $k$ to store the agent's index of its source. An example is shown in Fig. [5 where $m \in \mathcal{N}_{\ell}$ but $m \notin \mathcal{N}_{k}$. First, the anchor vectors and the source agents for agents $k$ and $\ell$ at time instant $i=0$ (Fig. [5(a)) are given, respectively, by

$$
\breve{\boldsymbol{\psi}}_{k, 0}=0, \boldsymbol{s}_{k}(0)=0, \breve{\boldsymbol{\psi}}_{\ell, 0}=0, \boldsymbol{s}_{\ell}(0)=0
$$

The anchor vectors and the source agents for agents $k$ and $\ell$ at time instants $i=\{1,2\}($ Fig. 5 (b) and (c)) are given, respectively, by

$$
\begin{aligned}
\breve{\boldsymbol{\psi}}_{k, 1} & =0, \boldsymbol{s}_{k}(1)=0, \breve{\boldsymbol{\psi}}_{\ell, 1}=\boldsymbol{\psi}_{m, 1}, \boldsymbol{s}_{\ell}(1)=m, \\
\breve{\boldsymbol{\psi}}_{k, 2} & =\breve{\boldsymbol{\psi}}_{\ell, 1}, \boldsymbol{s}_{k}(2)=\ell, \breve{\boldsymbol{\psi}}_{\ell, 2}=\boldsymbol{\psi}_{m, 2}, \boldsymbol{s}_{\ell}(2)=m .
\end{aligned}
$$

Agents update their anchor vectors $\left\{\breve{\boldsymbol{\psi}}_{k, i}\right\}$ at each time instant $i$ by the following step:

$$
\breve{\psi}_{k, i}= \begin{cases}\psi_{m, i}, & \text { if } m \in \mathcal{N}_{k}, \\ \breve{\psi}_{\ell, i-1}, & \text { if } \ell \in \mathcal{N}_{k} \text { and } \boldsymbol{s}_{k}(i)=0 \text { and } \boldsymbol{s}_{\ell}(i) \neq 0 \\ \breve{\psi}_{\ell, i-1}, & \text { if } \ell \in \mathcal{N}_{k} \text { and } \boldsymbol{s}_{k}(i)=\ell \\ \breve{\psi}_{k, i-1}, & \text { otherwise }\end{cases}
$$

where $\breve{\boldsymbol{\psi}}_{m, i}=\boldsymbol{\psi}_{m, i}$ for agent $m$ itself. The source of the anchor vector is 
updated simultaneously as follows:

$$
\boldsymbol{s}_{k}(i)= \begin{cases}m, & \text { if } m \in \mathcal{N}_{k}, \\ \ell, & \text { if } \boldsymbol{s}_{k}(i)=0 \text { and } \boldsymbol{s}_{\ell}(i) \neq 0 \\ \boldsymbol{s}_{k}(i-1), & \text { otherwise }\end{cases}
$$

Similarly to the previous section, the next step is to update the coefficients $\left\{\boldsymbol{h}_{\ell k}(i)\right\}$ using the following test:

$$
\boldsymbol{h}_{\ell k}(i)= \begin{cases}1, & \text { if } \boldsymbol{s}_{\ell}(i) \neq 0 \text { and } \boldsymbol{s}_{k}(i) \neq 0, \\ 0, & \text { otherwise. }\end{cases}
$$

Again, having $\boldsymbol{s}_{k}(i) \neq 0$ leads to the situation that agent $k$ has the anchor vector and has been informed about the decision of the network, therefore, agent $k$ can start sharing information with the other agents whose $\boldsymbol{s}_{\ell}(i) \neq 0$ as well to estimate the desired model. The matrix $\boldsymbol{G}_{i}$ will be generated using (20).

Agents update the coefficients of both matrices $\dot{A}_{i}$ and $\ddot{A}_{i}$ using the following steps:

$$
\begin{aligned}
& \dot{\boldsymbol{a}}_{\ell k}(i)= \begin{cases}\boldsymbol{g}_{\ell k}(i), & \text { if }\left\|\breve{\boldsymbol{\psi}}_{k, i}-\boldsymbol{\psi}_{\ell, i}\right\|^{2} \leq \beta, \\
0, & \text { otherwise. }\end{cases} \\
& \ddot{\boldsymbol{a}}_{\ell k}(i)= \begin{cases}\boldsymbol{g}_{\ell k}(i), & \text { if } \dot{\boldsymbol{a}}_{\ell k}(i)=0, \\
0, & \text { otherwise. }\end{cases}
\end{aligned}
$$

Then, the estimate $\boldsymbol{w}_{k, i}$ is updated using Eq. (12). According to (34) and (12), agent $k$ combines $\phi_{\ell, i}$ if the desired model of the network (which is represented by the anchor vector $\breve{\psi}_{k, i}$ of agent $k$ ) is close to the observed model of agent $\ell$ that is represented by $\boldsymbol{\psi}_{\ell, i}$. Algorithm 2 summarizes the steps of the approach for following the observed model of a specific agent $m$.

\section{Simulation Results and Discussion}

\subsection{Static Network}

We consider a connected network with 80 randomly distributed agents. The agents observe data originating from $C=3$ different models. Each model 


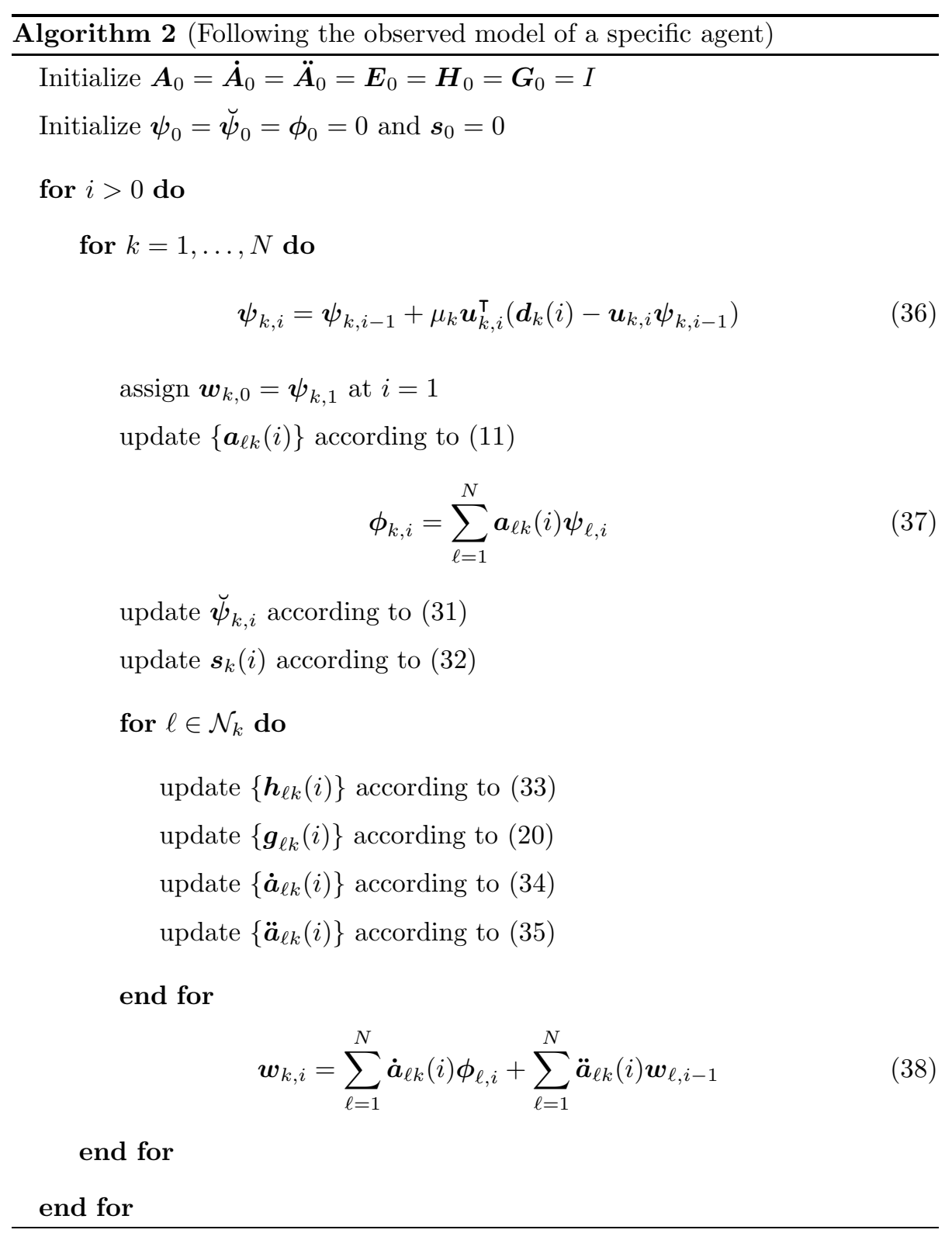


(a)

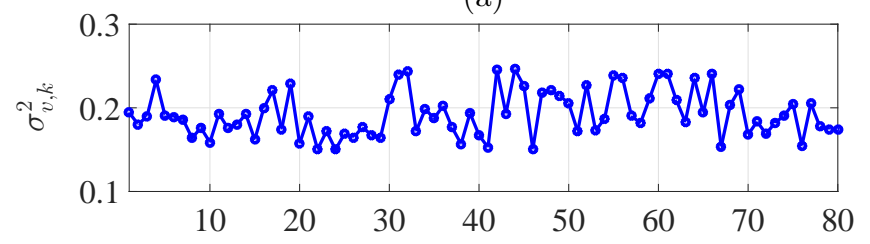

(b)

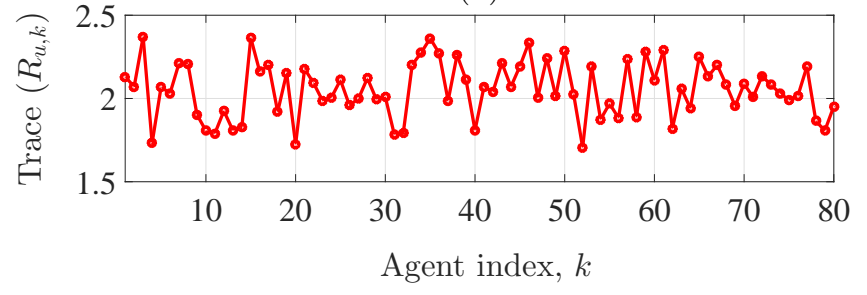

Figure 6: Statistical noise and signal profiles over the network.

$z_{j}^{\circ} \in \mathbb{R}^{M \times 1}$ is generated as follows: $z_{j}^{\circ}=\left[r_{1}, \ldots, r_{M}\right]^{\top}$ where $r_{m} \in[1,-1]$, $M=2$. The assignment of the agents to models is random. The maximum number of neighbors is $n_{k}=7$. We set $\{\alpha, \beta, \nu, \mu\}=\{0.04,0.08,0.005,0.01\}$. We use the uniform combination policy to generate the coefficients $\left\{\boldsymbol{a}_{\ell k}(i)\right\}$ and $\left\{\boldsymbol{g}_{\ell k}(i)\right\}$.

Figure 6 shows the statistical profile of the regressors and noise across the agents. The regressors are of size $M=2$ zero-mean Gaussian, independent in time and space, and have diagonal covariance matrices $R_{u, k}$. Figure 7 shows the topology of one of 100 Monte Carlo experiments. Agents having the same inner color observe the same model, while the outer color indicates the desired model at steady-state.

The transient network mean-square deviation (MSD) regarding each observed model $z_{j}^{\circ}$ at each time instant $i$ is defined by

$$
\operatorname{MSD}_{j}(i) \triangleq \frac{1}{\left|\mathcal{C}_{j}\right|} \sum_{k \in \mathcal{C}_{j}}\left\|z_{j}^{\circ}-\phi_{k, i}\right\|^{2}
$$

where $j=1, \ldots, C$ and each $\mathrm{MSD}_{j}$ is computed for agents belonging to $\mathcal{C}_{j}$. The transient network mean-square deviation (MSD) for the whole network 
(a)

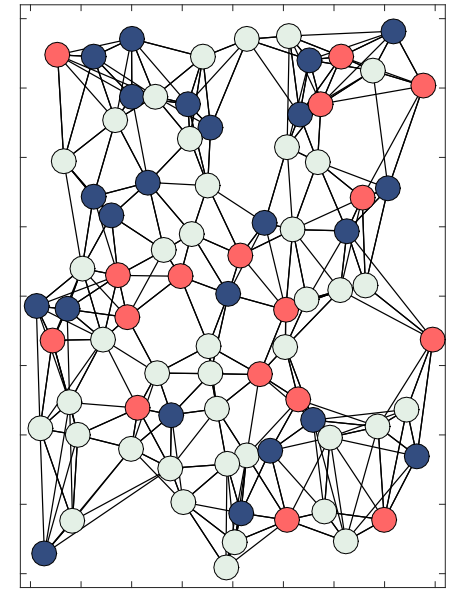

(b)

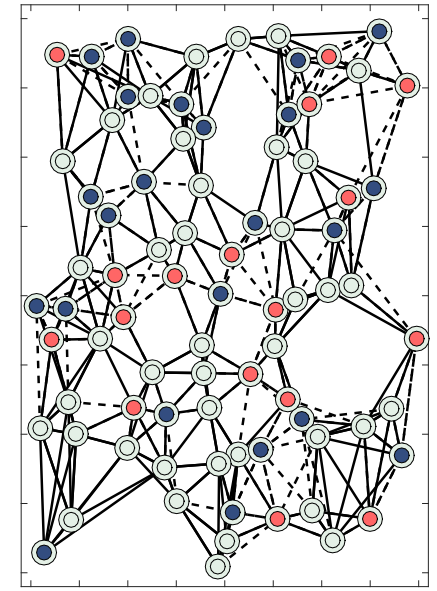

Figure 7: Network topology (a) and final decision of the agents where the bold (dashed) links represent $\{\dot{\boldsymbol{a}}(i)\}(\{\ddot{\boldsymbol{a}}(i)\})$ at steady-state (b).

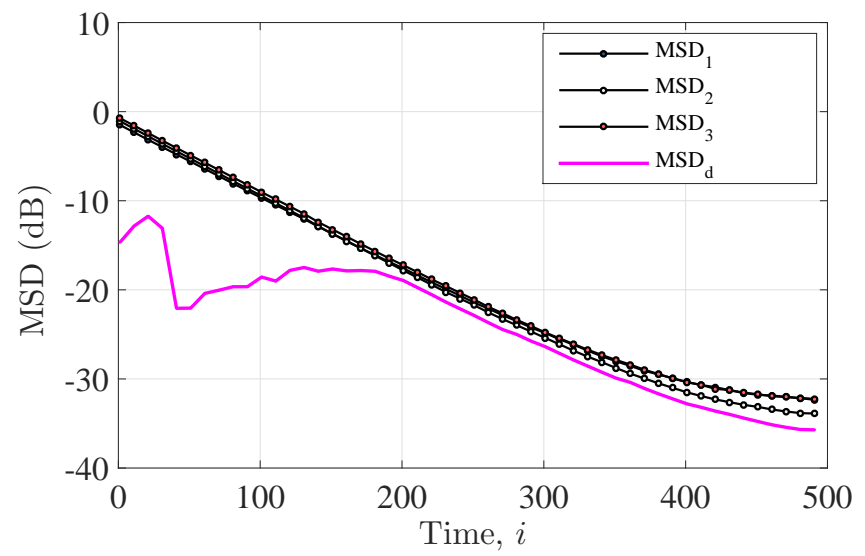

Figure 8: Transient mean-square deviation (MSD). 
(a)

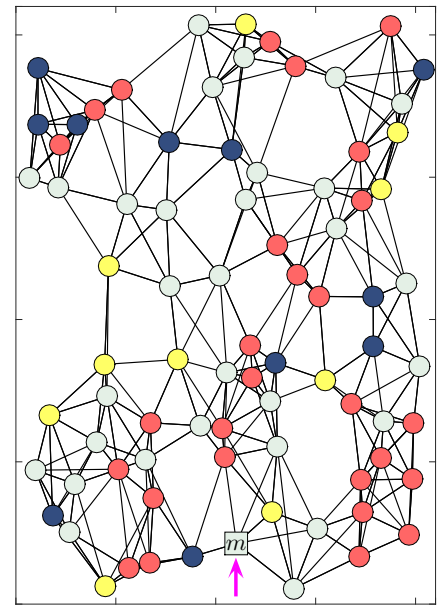

(b)

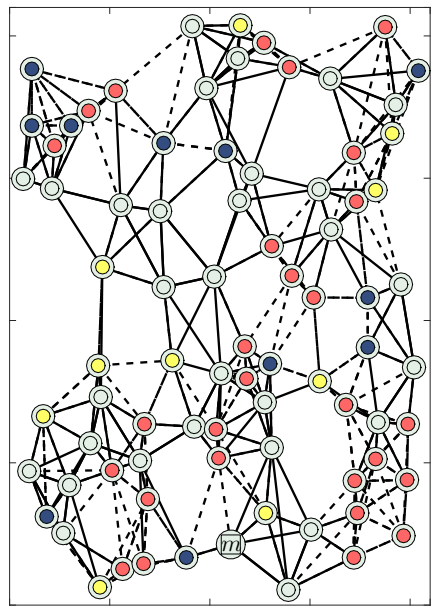

Figure 9: Network topology (a) and final decision of the agents to follow the model of agent $m$ where the bold (dashed) links represent $\{\dot{\boldsymbol{a}}(i)\}(\{\ddot{\boldsymbol{a}}(i)\})$ at steady-state (b).

regarding the desired model at each time instant $i$ is defined by

$$
\operatorname{MSD}_{\mathrm{d}}(i) \triangleq \frac{1}{N} \sum_{k=1}^{N}\left\|z_{d}^{\circ}-\boldsymbol{w}_{k, i}\right\|^{2}
$$

where $z_{d}^{\circ}$ is the desired model when the whole network agrees on one common desired model, i.e., $\operatorname{MSD}_{\mathrm{d}}(i)$ is only computed at the instants when all $\left\{\boldsymbol{p}_{k}(i)\right\}=1$. Figure 8 depicts the simulated transient mean-square deviation (MSD) of the network for all observed models and for the network desired model. Table1 displays the success rate of the decision-making to agree on one model for different numbers of observed models, $C \in\{2,3,4,5\}$. The proposed strategy appears to provide good success rate under the simulated conditions.

Table 1 Decision-making success rate for different $C$.

\begin{tabular}{c|c|c|c|c|}
$C$ & 2 & 3 & 4 & 5 \\
\hline Success rate & $99 \%$ & $98 \%$ & $99 \%$ & $99 \%$
\end{tabular}

Regarding the application of following the observed model of a specific agent $m$, Fig. 9 shows the topology of one case from 100 different experiments. Agents 


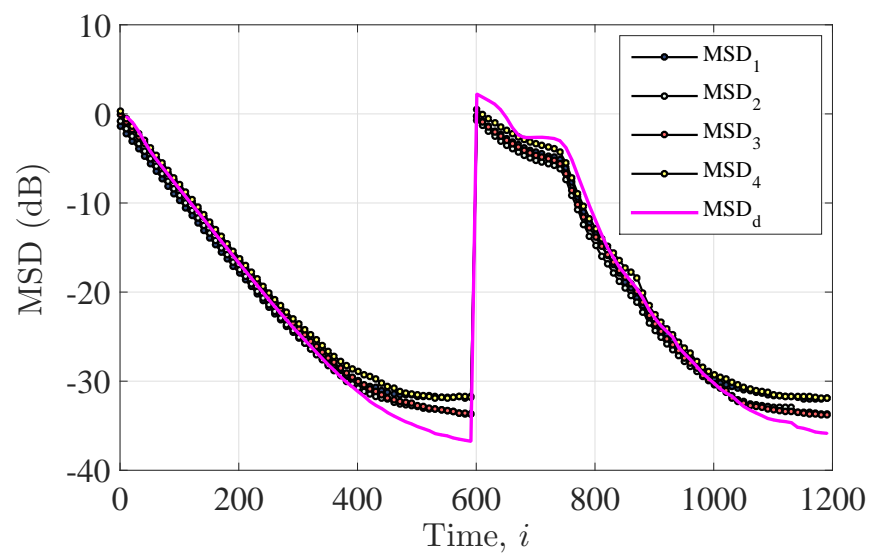

Figure 10: Transient mean-square deviation (MSD).

are observing $C=4$ different models. Agent $m=10$, which is represented by a square, is the specific agent whose observed model the whole network wishes to follow. Figure 10 shows the transient mean-square deviation MSD of 100 different experiments when a change in the model assignment occurs suddenly at time instant $i=600$. The success rate of the decision-making to agree on the observed model of agent $m$ was observed to be $100 \%$ in this simulation.

\subsection{Mobile Network}

We consider a network with 80 randomly distributed mobile agents [2]. The agents observe data originating from four different models (sources) $C=4$, where $w_{r_{m}} \in[50,-50]$. The objective of the network is to have all agents track and move towards only one model (source). Figure 11 shows the statistical profile of the regressors and noise across the agents. Every agent $k$ updates its location according to the motion mechanism described in [19].

Figure 12 shows the maneuver of the agents over time where the models (sources) are represented by squares. Figure 13 represents the transient network mean-square deviation (MSD) obtained by averaging over 100 independent Monte Carlo experiments. 
(a)

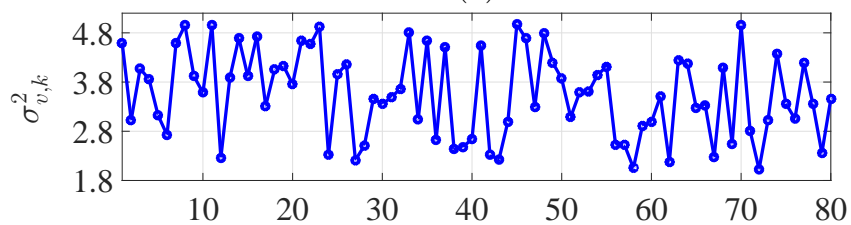

(b)

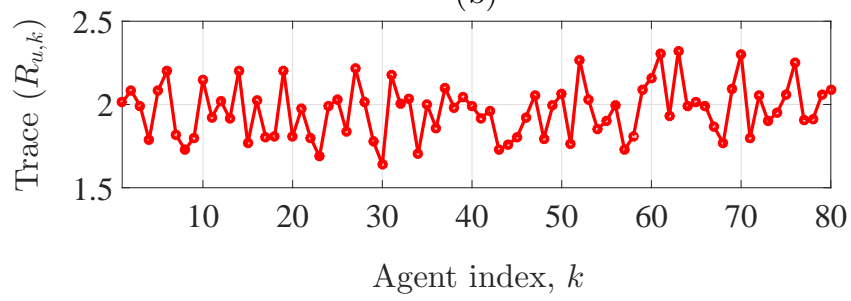

Figure 11: Statistical noise and signal profiles over the mobile network.
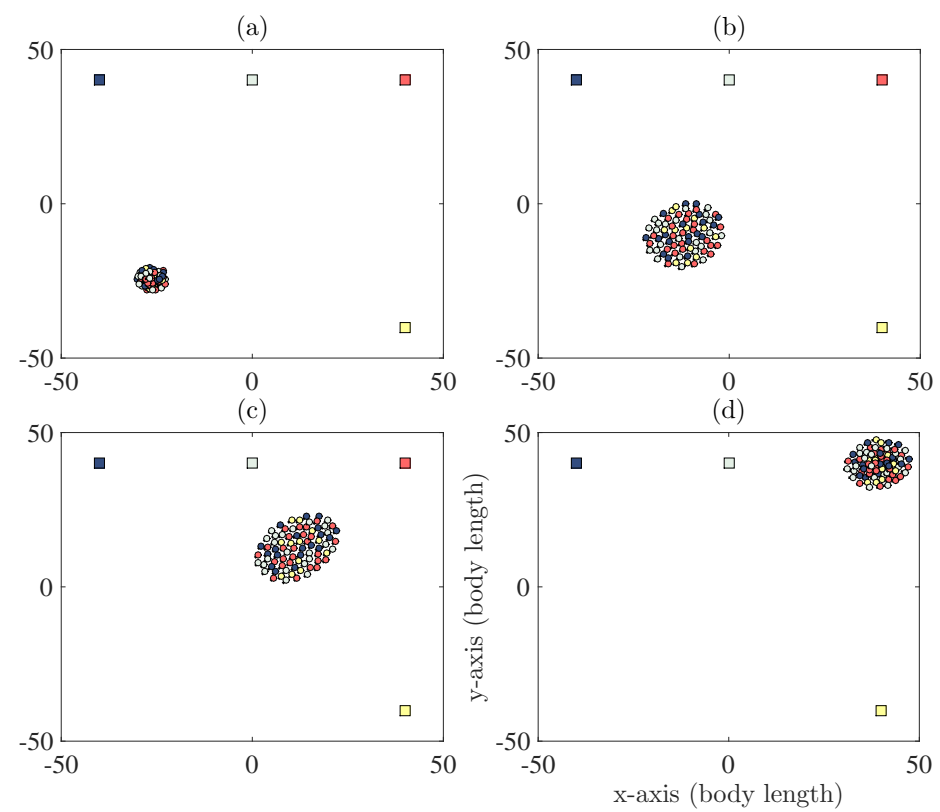

Figure 12: Maneuver of the agents with four sources over time (a) $i=1$, (b) $i=200$, (c) $i=500$, and (d) $i=1000$. The unit length is the body length of a agent. 


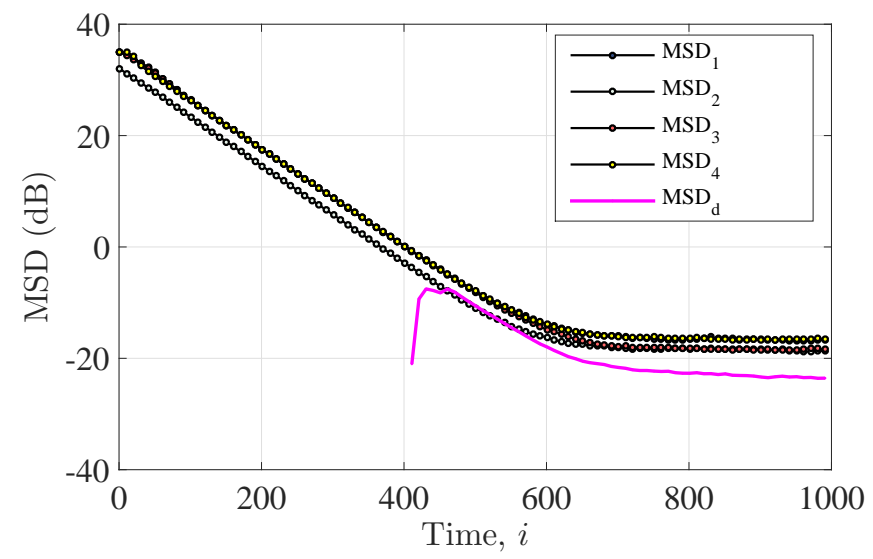

Figure 13: Transient mean-square deviation (MSD) of the mobile network.

\section{Conclusion}

We have proposed a distributed algorithm that allows agents over multi-task networks to follow only one common model while proceeding with the estimation process. Agents use a local labeling step to distinguish the multiple desired models of their neighbors. Simulation results illustrate the operation of the algorithms and its performance.

\section{Acknowledgements}

The research for this paper was financially supported by Technische Universität Darmstadt, Signal Processing Group and NSF grant CCF-1524250.

\section{References}

\section{References}

[1] F. S. Cattivelli, A. H. Sayed, Modeling bird flight formations using diffusion adaptation, IEEE Trans. Signal Process. 59 (5) (2011) 2038-2051.

[2] S. Y. Tu, A. H. Sayed, Mobile adaptive networks, IEEE J. Sel. Topics Signal Process. 5 (4) (2011) 649-664. 
[3] A. Avitabile, R. A. Morse, R. Boch, Swarming honey bees guided by pheromones, Ann. Entonomol. Soc. Am. 68 (1975) 1079-1082.

[4] H. Berg, Motile behavior of bacteria, Physics Today 53 (1) (2000) 24-29.

[5] F. Dressler, O. B. Akan, Bio-inspired networking: from theory to practice, IEEE Communications Magazine (2010) 176-183.

[6] S. Camazine, J. L. Deneubourg, N. R. Franks, G. T. J. Sneyd, E. Bonabeau, Self-Organization in Biological Systems, Princeton, NJ: Princeton Univ. Press, 2003.

[7] J. C. X. Z. A. H. Sayed, S.Y. Tu, Z. Towfic, Diffusion strategies for adaptation and learning over networks: an examination of distributed strategies and network behavior, IEEE Signal Processing Magazine 30 (3) (2013) $155-171$.

[8] A. H. Sayed, Adaptation, learning, and optimization over networks, Found. Trends in Mach. Learn. 7 (4-5) (2014) 311-801.

[9] X. Zhao, A. H. Sayed, Clustering via diffusion adaptation over networks, in: Proc. International Workshop on Cognitive Inform. Processing (CIP), Baiona, Spain, 2012, pp. 1-6.

[10] J. Chen, C. Richard, A. H. Sayed, Diffusion LMS over multitask networks, IEEE Trans. Signal Processing 63 (11) (2015) 2733-2748.

[11] X. Zhao, A. H. Sayed, Distributed clustering and learning over networks, IEEE Trans. Signal Processing 63 (13) (2015) 3285-3300.

[12] S. Khawatmi, A. M. Zoubir, Decentralized partitioning over adaptive networks, in: Proc. IEEE International Workshop on Machine Learning for Signal Processing (MLSP), Vietri sul Mare, Salerno, Italy, 2016, pp. 1-6. doi:10.1109/MLSP.2016.7738880 
[13] M. Z. Lin, M. N. Murthi, K. Premaratne, Mobile adaptive networks for pursuing multiple targets, in: Proc. IEEE Int. Conf. Acoust. Speech Signal Process. (ICASSP), South Brisbane, QLD, 2015, pp. 3217-3221.

[14] M. Hajiabadi, H. Khoshbin, G. A. Hodtani, Transient performance analysis of adaptive multitask network based on correntropy criterion, International Journal of Adaptive Control and Signal Processing 32 (2) (2018) 229-247.

[15] C. Zhang, P. Zhao, S. Hao, Y. C. Soh, B. S. Lee, C. Miao, S. C. H. Hoi, Distributed multi-task classification: a decentralized online learning approach, Machine Learning 107 (4) (2018) 727-747.

[16] D. Acemoglu, A. Ozdaglar, Opinion dynamics and learning in social networks, Dynamic Games and Applications 1 (1) (2011) 3-49.

[17] S. P. Talebi, S. Werner, V. Koivunen, Kalman filtering and clustering in sensor networks, in: 2018 IEEE International Conference on Acoustics, Speech and Signal Processing (ICASSP), IEEE, 2018, pp. 4309-4313.

[18] S. Y. Tu, A. H. Sayed, Distributed decision-making over adaptive networks, IEEE Trans. Signal Processing 62 (5) (2014) 1054-1069.

[19] S. Khawatmi, X. Huang, A. M. Zoubir, Distributed decision-making over mobile adaptive networks, in: Proc. IEEE Int. Conf. Acoust. Speech Signal Process. (ICASSP), New Orleans, USA, 2017, pp. 3864-3868.

[20] S. Khawatmi, A. H. Sayed, A. M. Zoubir, Decentralized clustering and linking by networked agents, IEEE Trans. Signal Processing 65 (13) (2017) $3526-3537$. 